CERN-TH/95-18

DESY 95-039

IEM-FT-97/95

hep-ph/9504241

\title{
Improved metastability bounds on the Standard Model Higgs mass "艹
}

\author{
J.R. Espinosa [ \\ Deutsches Elektronen-Synchrotron DESY, Hamburg, Germany \\ and \\ M. Quirós f \\ CERN, TH Division, CH-1211 Geneva 23, Switzerland
}

\begin{abstract}
Depending on the Higgs-boson and top-quark masses, $M_{H}$ and $M_{t}$, the effective potential of the Standard Model at finite (and zero) temperature can have a deep and unphysical stable minimum $\langle\phi(T)\rangle$ at values of the field much larger than $G_{F}^{-1 / 2}$. We have computed absolute lower bounds on $M_{H}$, as a function of $M_{t}$, imposing the condition of no decay by thermal fluctuations, or quantum tunnelling, to the stable minimum. Our effective potential at zero temperature includes all next-to-leading logarithmic corrections (making it extremely scale-independent), and we have used pole masses for the Higgs-boson and top-quark. Thermal corrections to the effective potential include plasma effects by one-loop ring resummation of Debye masses. All calculations, including the effective potential and the bubble nucleation rate, are performed numerically, and so the results do not rely on any kind of analytical approximation. Easy-to-use fits are provided for the benefit of the reader. Conclusions on the possible Higgs detection at LEP-200 are drawn.
\end{abstract}

CERN-TH/95-18

March 1995

*Work supported in part by the European Union (contract CHRX-CT92-0004) and CICYT of Spain (contract AEN94-0928).

†Supported by Alexander-von-Humboldt Stiftung.

$\ddagger$ On leave of absence from Instituto de Estructura de la Materia, CSIC, Serrano 123, 28006-Madrid, Spain. 


\section{Introduction}

For a particular range of values of the Higgs-boson and top-quark masses, $M_{H}$ and $M_{t}$, the effective potential of the Standard Model (SM) exhibits an unphysical stable minimum at values of the field much larger than the electroweak scale. This effect is accentuated for large top Yukawa coupling $h_{t}$, which drives the SM quartic coupling $\lambda$ to negative values at large scales. Therefore, the vacuum stability requirement in the SM imposes a severe lower bound on $M_{H}$, which depends on $M_{t}$ and the cutoff $\Lambda$ beyond which new physics operates. This bound was computed in various approximations [1-5], and, more recently, using the improved one-loop effective potential including all nextto-leading logarithm corrections and pole masses for the Higgs-boson and the top-quark [6-8]. It was proved in ref. [8] that the latter effects can be very specially important, in particular for large top-quark masses (as the recent experimental evidence indicates [9, 10]) and for low values of $\Lambda$ (which can be interesting for the future range of masses that will be covered at LEP-200 [11]).

However, even if the lower bounds on $M_{H}$ arising from stability requirements are a valuable indication, they cannot be considered as absolute lower bounds in the SM since we cannot logically exclude the possibility of the physical electroweak minimum being a metastable one, provided the probability, normalized with respect to the expansion rate of the Universe, for decay to the unphysical (true) minimum, be negligibly small. This we will call metastability requirement. A first step in that direction was given in ref. [12], where bounds on the Higgs mass from the requirement of metastability of the electroweak vacuum at finite temperature, for temperatures below the critical temperature of the electroweak phase transition, were given. It was subsequently noticed [13] that the strongest bounds come from the requirement of metastability for temperatures higher than the electroweak critical temperature. In that paper the effective potential was calculated in the leading-logarithm approximation, with tree-level masses for the Higgs-boson and top-quark, and using the high-temperature limit for thermal corrections as well as semi-analytical approximations for the calculation of the energy of the critical bubble and so the tunnelling probability by thermal fluctuations.

In view of the future Higgs search at LEP-200 and future colliders, it is extremely important that the bounds provided on the Higgs mass in the SM be as accurate as possible. In this paper we will compute metastability lower bounds on the Higgs mass as a function of the top mass and the cutoff $\Lambda$ using:

- an effective potential including next-to-leading logarithm corrections, and guaranteeing to a large extent scale independence, as in [8];

- physical (pole) masses for the Higgs-boson and top-quark;

- thermal corrections to the effective potential including plasma effects by one-loop resummation of Debye masses; these corrections are evaluated numerically and thus do not rely on the high temperature expansion;

- numerical calculation of the bounce solution and the energy of the critical bubble. 
As a consequence of the previous input, our lower bound on $M_{H}$ reduces dramatically with respect to the results of ref. [13]. To fix the ideas, for $M_{t}=175 \mathrm{GeV}$, the bound reduces by $\sim 10 \mathrm{GeV}$ for $\Lambda=10^{4} \mathrm{GeV}$, and $\sim 30 \mathrm{GeV}$ for $\Lambda=10^{19} \mathrm{GeV}$.

\section{The effective potential}

The starting point in our analysis is the effective potential of the SM at finite temperature. It contains the usual zero-temperature term and the thermal corrections [14] as:

$$
V_{\text {eff }}(\phi, T)=V_{\text {eff }}(\phi, 0)+\Delta V_{\text {eff }}(\phi, T) .
$$

The first term in (看) can be written in the 't Hooft-Landau gauge and the $\overline{\mathrm{MS}}$ scheme as [5]:

$$
V_{\text {eff }}(\phi, 0)=\sum_{\mathrm{L}} V_{\mathrm{L}}(\phi)
$$

where $V_{\mathrm{L}}$ is the L-loop correction to the effective potential at zero-temperature, namely

$$
\begin{gathered}
V_{0}=-\frac{1}{2} m^{2}(t) \phi^{2}(t)+\frac{1}{8} \lambda(t) \phi^{4}(t) \\
V_{1}=\sum_{i=W, Z, t} \frac{n_{i}}{64 \pi^{2}} m_{i}^{4}(\phi)\left[\log \frac{m_{i}^{2}(\phi)}{\mu^{2}(t)}-C_{i}\right]+\Omega(t),
\end{gathered}
$$

where

$$
n_{W}=6, \quad n_{Z}=3, \quad n_{t}=-12, \quad C_{W}=C_{Z}=\frac{5}{6}, \quad C_{t}=\frac{3}{2},
$$

the masses are defined as usual by

$$
m_{W}^{2}(\phi)=\frac{1}{4} g^{2}(t) \phi^{2}(t), \quad m_{Z}^{2}(\phi)=\frac{1}{4}\left(g^{2}(t)+g^{\prime 2}(t)\right) \phi^{2}(t), \quad m_{t}^{2}(\phi)=\frac{1}{2} h_{t}^{2}(t) \phi^{2}(t),
$$

and $\Omega$ is the one-loop contribution to the cosmological constant [5], which will turn out to be irrelevant in our calculation. Likewise the contribution of the Higgs and Goldstone boson sector can be consistently included [15], although it is numerically irrelevant.

In eqs. (3) and (4) the parameters $\lambda(t)$ and $m(t)$ are the SM quartic coupling and mass, whereas $g(t), g^{\prime}(t)$ and $h_{t}(t)$ are the $S U(2), U(1)$ and top Yukawa couplings respectively. All parameters are running with the SM renormalization group equations (RGE). The Higgs field is running as $\phi(t)=\xi(t) \phi$, with $\xi(t)=\exp \left\{-\int_{0}^{t} \gamma\left(t^{\prime}\right) d t^{\prime}\right\}$ where $\gamma(t)$ is the anomalous dimension of the Higgs field. Finally the scale $\mu(t)$ is related to the running parameter $t$ by $\mu(t)=\mu \exp (t)$, where $\mu$ is a given scale that fixes the starting of the running and will be taken equal to the physical $Z$ mass.

It has been shown [16] that the L-loop effective potential improved by $(\mathrm{L}+1)$ loop RGE resums all $\mathrm{L}_{\mathrm{th}}$-to-leading logarithm contributions. Consequently we have considered [8] all the $\beta$ - and $\gamma$-functions of the previous parameters to two-loop order [5] so that our calculation contains all next-to-leading logarithmic corrections.

The potential (2) has been proved [8, 15] to be very scale-independent. It means that any judicious choice of the scale $\mu(t)$ should give a very good and accurate numerical 
description of it. In particular the choice $\mu(t)=\phi(t)$ minimizes the size of radiative corrections and will be taken from here on. The potential (2) can have, depending on the values of the Higgs-boson and top-quark masses, other minima at large values of the field. In particular it was shown that at any stationary point of (2), $\phi_{\text {stat }}=2 m^{2} / \tilde{\lambda}$, with

$$
\begin{aligned}
\tilde{\lambda} & =\lambda-\frac{1}{16 \pi^{2}}\left\{6 h_{t}^{4}\left[\log \frac{h_{t}^{2}}{2}-1\right]-\frac{3}{4} g^{4}\left[\log \frac{g^{2}}{4}-\frac{1}{3}\right]\right. \\
& \left.-\frac{3}{8}\left(g^{2}+g^{\prime 2}\right)^{2}\left[\log \frac{\left(g^{2}+g^{\prime 2}\right)}{4}-\frac{1}{3}\right]\right\} .
\end{aligned}
$$

For stationary points much larger than the electroweak scale, $\tilde{\lambda} \ll 1$ and the curvature of the potential is given by

$$
\left.\frac{\partial^{2} V_{\text {eff }}}{\partial \phi^{2}(t)}\right|_{\phi(t)=\phi_{\text {stat }}(t)}=\frac{1}{2}\left(\beta_{\lambda}-4 \gamma \lambda\right) \phi_{\text {stat }}^{2} \simeq \frac{1}{2} \beta_{\lambda} \phi_{\text {stat }}^{2},
$$

where all the parameters are evaluated at the scale $\phi_{\text {stat }}$. Eq. (6) shows that the stationary point is a minimum (maximum) if $\beta_{\lambda}>0\left(\beta_{\lambda}<0\right)$.

In fact, the large field structure of the effective potential at zero-temperature can be understood from (6). Typically, for low scales $\beta_{\lambda}$ is negative and dominated by the term $-12 h_{t}^{4}$. This means that $\tilde{\lambda}$ is decreasing as the scale increases and will satisfy the condition $\tilde{\lambda} \sim 0$ for a stationary point, which will turn out to be a maximum since $\beta_{\lambda}$ is negative. However, as the top coupling $h_{t}$ decreases with the scale, and the gauge couplings provide a positive contribution to $\beta_{\lambda}$, at a given scale the contribution of top and gauge couplings will balance and there will be a turn over at a particular scale from negative to positive $\beta_{\lambda}$. This will make $\tilde{\lambda}$ to increase and cross zero, indicating the presence of a minimum, since now $\beta_{\lambda}>0$.

The previous pattern translates into a well-defined structure of the effective potential at zero-temperature. The locations of maxima and minima depend on the SM parameters, in particular on the Higgs-boson and top masses. Stability bounds can be established on the basis that the maximum J occurs for values of the field larger than the cutoff $\Lambda$ beyond which the SM is no longer valid.

Using pole (physical) values of the Higgs mass $M_{H}$ :

$$
M_{H}^{2}=m_{H}^{2}(t)+\operatorname{Re}\left[\Pi\left(p^{2}=M_{H}^{2}\right)-\Pi\left(p^{2}=0\right)\right],
$$

where $m_{H}(t)$ is the running mass defined as the second derivative of the effective potential and $\Pi\left(p^{2}\right)$ is the renormalized self-energy of the Higgs-boson 2 , and top-quark mass [17] $M_{t}$ :

$$
M_{t}=\left\{1+\frac{4}{3} \frac{\alpha_{S}\left(M_{t}\right)}{\pi}+\left[16.11-1.04 \sum_{i=1}^{5}\left(1-\frac{M_{i}}{M_{t}}\right)\right]\left(\frac{\alpha_{S}\left(M_{t}\right)}{\pi}\right)^{2}\right\} m_{t}\left(M_{t}\right),
$$

\footnotetext{
${ }^{1}$ Strictly speaking we should replace in this sentence "the maximum of the potential" by the "value of the field where the potential is deeper than the electroweak minimum". However the difference is numerically irrelevant given the sharp descent of the potential after the maximum.

${ }^{2}$ The one-loop $t$-dependence of $(7)$ drops out. Explicit expressions for $m_{H}^{2}(t)$ and $\Pi\left(M_{H}^{2}\right)-\Pi(0)$ can be found in ref. [15].
} 
where $M_{i}, i=1, \ldots, 5$, represent the masses of the five lighter quarks, lower bounds on $M_{H}$ as a function of $M_{t}$ and $\Lambda$ were put in [8]. We will later on compare these bounds with those that will be obtained in this paper. In Fig. 1 we show the shape of the effective potential at zero-temperature (thick solid line) for $M_{t}=175 \mathrm{GeV}$ and $M_{H} \sim 122 \mathrm{GeV}$. We see that there is a deep minimum at large values of $\phi$. Requiring absolute stability of the effective potential leads to a rejection of these values of $M_{t}$ and $M_{H}$, as can also be seen in 8$]$.

The thermal correction to Eq. (1) can be computed using the rules of field theory at finite temperature [18, 19]. Including plasma effects [14, 18, 19, 20] by one-loop ring resummation of Debye masse 3 , it can be written as

$$
\Delta V_{\text {eff }}(\phi, T)=V_{1}(\phi, T)+V_{\text {ring }}(\phi, T) .
$$

The first term in (9) is the one-loop thermal correction

$$
V_{1}(\phi, T)=\frac{T^{4}}{2 \pi^{2}}\left[\sum_{i=W, Z} n_{i} J_{B}\left(\frac{m_{i}^{2}(\phi)}{T^{2}}\right)+n_{t} J_{F}\left(\frac{m_{t}^{2}(\phi)}{T^{2}}\right)\right]
$$

where the functions $J_{B}$ and $J_{F}$ are defined by

$$
J_{B}(y)=\int_{0}^{\infty} d x x^{2} \log \left[1-e^{-\sqrt{x^{2}+y^{2}}}\right]
$$

and

$$
J_{F}(y)=\int_{0}^{\infty} d x x^{2} \log \left[1+e^{-\sqrt{x^{2}+y^{2}}}\right] .
$$

Plasma effects in the leading approximation can be accounted for by the one-loop effective potential improved by the daisy diagrams [18, 20]. This approximation takes into account the contribution of hard thermal loops in the higher-loop expansion. The second term of Eq. (9) is given by [21]

$$
V_{\text {ring }}(\phi, T)=\sum_{i=W_{L}, Z_{L}, \gamma_{L}} n_{i}\left\{\frac{m_{i}^{3}(\phi) T}{12 \pi}-\frac{\mathcal{M}_{i}^{3}(\phi) T}{12 \pi}\right\},
$$

where only the longitudinal degrees of freedom of gauge bosons,

$$
\frac{1}{2} n_{W_{L}}=n_{Z_{L}}=n_{\gamma_{L}}=1
$$

are accounted. The Debye-corrected masses are given by

$$
\begin{aligned}
\mathcal{M}_{W_{L}}^{2} & =m_{W}^{2}(\phi)+\frac{11}{6} g^{2} T^{2} \\
\mathcal{M}_{Z_{L}}^{2} & =\frac{1}{2}\left[m_{Z}^{2}(\phi)+\frac{11}{6} \frac{g^{2}}{\cos ^{2} \theta_{W}} T^{2}+\Delta(\phi, T)\right] \\
\mathcal{M}_{\gamma_{L}}^{2} & =\frac{1}{2}\left[m_{Z}^{2}(\phi)-\frac{11}{6} \frac{g^{2}}{\cos ^{2} \theta_{W}} T^{2}+\Delta(\phi, T)\right]
\end{aligned}
$$

\footnotetext{
${ }^{3}$ This approximation is good enough for our purposes in this paper, since the infrared problem for $\phi=0$ will not affect the phase transition to values of the field $\gg G_{F}^{-1 / 2}$.
} 
where the discriminant

$$
\Delta^{2}(\phi, T)=m_{Z}^{4}(\phi)+\frac{11}{3} \frac{\cos ^{2} 2 \theta_{W}}{\cos ^{2} \theta_{W}}\left[m_{Z}^{2}(\phi)+\frac{11}{12} \frac{g^{2}}{\cos ^{2} \theta_{W}} T^{2}\right] T^{2}
$$

is responsible for the rotation at finite temperature from the basis $\left(W_{3 L}, B_{L}\right)$ to the mass eigenstate basis $\left(Z_{L}, \gamma_{L}\right)$.

In Fig. 1 we plot the effective potential at $T=T_{t}=2.5 \times 10^{15} \mathrm{GeV}$ (thin solid line), for the previously considered values of $M_{t}$ and $M_{H}$, where the temperature $T_{t}$ will be defined in the next sections. We can see that for values of the field such that $\phi \lesssim 10 T_{t} \sim 10^{16} \mathrm{GeV}$ the thermal corrections dominate over the zero-temperature term, while for $\phi \gtrsim 10^{16} \mathrm{GeV}$, thermal corrections are exponentially suppressed and the potential drops to the zero-temperature value. For $T \gg T_{t}$ the minimum disappears and symmetry is restored.

\section{The thermal tunnelling}

In a first-order phase transition, such as that depicted in Fig. 1, the tunnelling probability rate per unit time per unit volume is given by 22, 23.

$$
\frac{\Gamma}{\nu} \sim \omega T^{4} e^{-E_{b} / T}
$$

where, for our purposes the prefactor $\omega$ can be taken to be $\mathcal{O}(1)$ as will be explained later, and $E_{b}$ (the energy of a bubble of critical size) is given by the three-dimensional euclidean action $S_{3}$ evaluated at the bounce solution $\phi_{B}$

$$
E_{b}=S_{3}\left[\phi_{B}(r)\right]
$$

At very high temperature the bounce solution has $O(3)$ symmetry, and the euclidean action is provided by

$$
S_{3}=4 \pi \int_{0}^{\infty} r^{2} d r\left[\frac{1}{2}\left(\frac{d \phi}{d r}\right)^{2}+V_{\mathrm{eff}}(\phi(r), T)\right]
$$

where $r^{2}=\vec{x}^{2}$, the potential is normalized as $V_{\text {eff }}(0, T)=0$, and the bounce $\phi_{B}$ satisfies the Euclidean equation of motion

$$
\frac{d^{2} \phi}{d r^{2}}+\frac{2}{r} \frac{d \phi}{d r}=\frac{d V_{\mathrm{eff}}(\phi, T)}{d \phi}
$$

with the boundary conditions H $^{-1}$

$$
\begin{aligned}
\lim _{r \rightarrow \infty} \phi(r) & =0 \\
\left.\frac{d \phi}{d r}\right|_{r=0} & =0
\end{aligned}
$$

\footnotetext{
${ }^{4}$ For $T<T_{c}^{\mathrm{EW}}$ the first condition in (20) should be replaced by $\phi(\infty)=v^{\mathrm{EW}}(T)$.
} 
The semiclassical picture is that unstable bubbles (either expanding or collapsing) are nucleated behind the barrier, at $\phi_{B}(0)$, with a probability rate given by (16). Whether or not they fill the Universe depends on the relation between the probability rate (16) and the expansion rate of the Universe. The actual probability $P$ is obtained by multiplying the probability rate (16) by the volume of our current horizon scaled back to the temperature $T$ and by the time the Universe spent at temperature $T$ [12]. One then obtains

$$
\frac{d P}{d \log T}=\kappa \frac{M_{P \ell}}{T} e^{-E_{b} / T}
$$

where

$$
\kappa \sim 3.25 \times 10^{86} .
$$

The total integrated probability is defined as

$$
P\left(T_{c}\right)=\int_{0}^{T_{c}} \frac{d P\left(T^{\prime}\right)}{d T^{\prime}} d T^{\prime},
$$

where $T_{c}$ is the temperature at which the two minima of the effective potential become degenerate. In fact, when $T \rightarrow T_{c}$ the probability rate goes to zero, since $E_{b}(T) \rightarrow \infty$.

Let us notice that the total probability $P \equiv P\left(T_{c}\right)$ is not normalized to unity. In fact the physical meaning of the integrated probability was discussed in ref. [25], where it was shown that the fraction of space in the (old) metastable phase in a first-order phase transition is given by

$$
f_{\text {old }}=e^{-P}
$$

and so the fraction of space in the (new) stable phase is

$$
f_{\text {new }}=1-e^{-P} .
$$

In this way for values of $P \ll 1$ all the space is in the metastable phase, while for $P \gg 1$ all the space is in the stable phase. We will see that the critical value of the probability, $P=\mathcal{O}(1)$, can simply be taken as the condition for the space to be in the metastable phase since, as a function of $M_{H}, P$ is very rapidly (exponentially) varying.

In Figs. 2 and 3 we show plots of $E_{b}$ and $d P / d \log _{10} T$, respectively, versus $\log _{10} T$ for $M_{t}=175 \mathrm{GeV}$ and $M_{H} \sim 122 \mathrm{GeV}$, as in Fig. 1. We see that there is a minimum of $E_{b} / T$ for a temperature of $\log _{10}(T / \mathrm{GeV}) \sim 17.5$ and, correspondingly, a maximum of $d P / d \log _{10} T$ for $\log _{10}(T / \mathrm{GeV}) \sim 16.5$. The fact that the two stationary points do not coincide is a consequence of the prefactor in (21), and proves that integrating (21) by the steepest-descent method around the point $B^{\prime}(T)=0, B(T) \equiv E_{b}(T) / T$, as can usually be found in the literature [25], is not a good approximation in our case. We have therefore performed the integral of (21) numerically. In Fig. 4 we have plotted the detail of the effective potential for the same values of $M_{t}$ and $M_{H}$ and the temperature $T_{t}$. We have shown, with the arrow on the tip of the wavy line, the location of the bounce solution $\phi_{B}(0) \sim 1.9 \times 10^{16} \mathrm{GeV}$ at this temperature.

As we can see from Fig. 3, the total integrated probability for the considered values of $M_{t}$ and $M_{H}$ is greater than 1. However, it may also happen to be smaller than 1. This will be the case for any fixed value of $M_{t}$ and sufficiently large values of $M_{H}$. For those values of $M_{t}$ and $M_{H}$, even if there is a metastable minimum at the origin, there 
is no dangerous transition to the deep stable minimum: in spite of appearances, the $\mathrm{SM}$ is robust for those values of $M_{t}$ and $M_{H}$. If, for the same value of $M_{t}$, we decrease the value of $M_{H}$, then the total integrated probability increases, until it reaches values of $\mathcal{O}(1)$ in which case $f_{\text {new }} \sim 1$ and the phase transition takes place.

The example that have been worked out in Figs. 1-4 have been tuned to get the bound on $M_{H}$ for $M_{t}=175 \mathrm{GeV}$. For $M_{H} \lesssim 122 \mathrm{GeV}$ the integrated probability increases and the phase transition always takes place. But, how sensitive is the obtained bound on $M_{H}$ to the precise definition of the (critical) probability for the onset of the phase transition? The answer is, very little. This is illustrated in Fig. 5 where we plot $\log P$, as a function of $M_{H}$ for $M_{t}=175 \mathrm{GeV}$. We can see that $\log P$ crosses zero at $M_{H} \sim 122 \mathrm{GeV}$, while $\Delta M_{H} \sim \pm 1 \mathrm{GeV}$ corresponds to $\Delta \log P \sim \pm 20$, which means that the error induced by the precise definition of the critical probability is negligible. This also means that no precise knowledge of the prefactor $\omega$ is required to get an accurate value of the bound on $M_{H}$. In fact, it has been shown [24] (computing the Higgs fluctuations on the background of the critical bubble) that for the electroweak phase transition the prefactor $\omega$ might significantly suppress the tunnelling rate. In our case the quartic Higgs coupling in the relevant range of scales is very small and these fluctuations will have no such a dramatic effect .

\section{The bounds}

In the previous section we have worked out in detail the bound on the Higgs mass $\left(M_{H} \gtrsim 122 \mathrm{GeV}\right)$ for a particular value of the top-quark mass $\left(M_{t}=175 \mathrm{GeV}\right)$ assuming implicitly a SM cutoff at a scale equal to $10^{19} \mathrm{GeV}$ (see Fig. 5). However the bounds should also depend on the actual value assumed for $\Lambda$.

We start from values of $M_{t}$ and $M_{H}$ such that $P=1$ in (23) for $\Lambda=10^{19} \mathrm{GeV}$. [This is, e.g., the case illustrated in Fig. 5 for $M_{t}=175 \mathrm{GeV}$ and $M_{H} \sim 122 \mathrm{GeV}$.] Keeping now $M_{t}$ fixed, $P$ increases when $M_{H}$ decreases. [See again Fig. 5 for illustration.] This means that for those values of $M_{H}$, and $\Lambda=10^{19} \mathrm{GeV}$, the phase transition should take place, as expected. However, for any different fixed value of $\Lambda, \Lambda<10^{19} \mathrm{GeV}$, we have to cutoff the integral (23) such that $\phi_{B}(0)$ at the maximal integration temperature $T_{t}$ satisfies 0

$$
\phi_{B}(0) \sim \Lambda
$$

The phase transition will take place for those values of $M_{H}$ such that

$$
P\left(T_{t}\right) \gtrsim 1
$$

In particular saturation of condition (27) leads to the actual bound on $M_{H}$ for $M_{t}$ and $\Lambda$ fixed.

From Figs. 1 and 4, and the results described in the previous section, we deduce that the obtained bound of $M_{H} \sim 122 \mathrm{GeV}$ for $M_{t}=175 \mathrm{GeV}$ corresponds to a

\footnotetext{
${ }^{5}$ Even ignoring this fact, the results of 24 give $\log \omega \sim-23$ (when the height of the barrier is small compared with the free-energy difference between the minima, as in our case), and Fig. 5 shows that this effect would change the bound on $M_{H}$ by less than $1 \mathrm{GeV}$.

${ }^{6}$ In fact Eq. (26) is our definition of $T_{t}$.
} 
maximal temperature of $T_{t}=2.5 \times 10^{15} \mathrm{GeV}$ (see Fig. 3) and a corresponding bounce of $\phi_{B}(0) \sim 10^{16} \mathrm{GeV}$ and a cutoff scale of $\Lambda \sim 10^{16} \mathrm{GeV}$. Moving down with $\Lambda$, and keeping $M_{t}$ fixed, one should move down with $M_{H}$ to saturate condition (26). This behaviour is illustrated in Fig. 6, where we plot the lower bound on $M_{H}$ as a function of $\Lambda$ for fixed values of $M_{t}$ from $140 \mathrm{GeV}$ to $200 \mathrm{GeV}$, and $\alpha_{S}\left(M_{Z}\right)=0.124$. The upper curve corresponds to $M_{t}=200 \mathrm{GeV}$ and the lower curve to $M_{t}=140 \mathrm{GeV}$. From Fig. 6 we can see that a measurement of $M_{H}$ and $M_{t}$ could give under certain circumstances, an upper bound on the scale of new physics. Given the present LEP bound on $M_{H}$ [26], $M_{H}>64.3 \mathrm{GeV}$ (95\% c.l.), only if $M_{t}>150 \mathrm{GeV}$ we could obtain an upper bound on the scale of new physics from the Higgs detection and mass measurement. For instance if we fix $M_{t}=200 \mathrm{GeV}$ we will obtain an upper bound on $\Lambda$ provided that $M_{H}<175$ $\mathrm{GeV}$. We will comment briefly, in the next section, on the implications of this fact for the Higgs search at LEP-200.

In Fig. 7 we have plotted the lower bound on $M_{H}$ as a function of $M_{t}$ for different values of $\Lambda$ : from $\log _{10}[\Lambda / \mathrm{GeV}]=4$ (lower solid) to 19 (upper solid). We also present in Fig. 7, for the sake of comparison, the lower bounds arising from the absolute stability requirements [8] for $\log _{10}[\Lambda / \mathrm{GeV}]=3$ (lower dashed), 4 and 19 (upper dashed). We can see that the solid curve corresponding to $\log _{10}[\Lambda / \mathrm{GeV}]=3$ has disappeared, which means that for this value of $\Lambda$, even if the electroweak minimum can be metastable (this corresponds to the region below the lower dashed line), it never decays into the stable unphysical minimum. The modification that the metastability bounds impose on the picture where only absolute stability was imposed can be easily traced back from Fig. 7. For $\Lambda=10^{19} \mathrm{GeV}$ it is negligible for $M_{t}=200 \mathrm{GeV}$, while it can be as large as $25 \mathrm{GeV}$ for $M_{t}=140 \mathrm{GeV}$. However, for small values of $\Lambda$ the modification is dramatic for the interesting range of $M_{t}$ considered. For instance, for $\Lambda=10^{4} \mathrm{GeV}$ the difference is $\sim 30 \mathrm{GeV}$ for $M_{t}=200 \mathrm{GeV}$ and $\sim 50 \mathrm{GeV}$ for $M_{t}=165 \mathrm{GeV}$.

We have made a linear fit to the solid curves of Fig. 7 as:

$$
M_{H} / \mathrm{GeV}=A(\Lambda)\left(M_{t} / \mathrm{GeV}\right)-B(\Lambda),
$$

where the coefficients $A$ and $B$ are given in the table. We have taken $\alpha_{S}\left(M_{Z}\right)=0.124$ and the fit is accurate to $1 \mathrm{GeV}$, for $M_{H}>60 \mathrm{GeV}$, i.e. $150 \mathrm{GeV}<M_{t}<200 \mathrm{GeV}$.

\begin{tabular}{||c|c|c||}
\hline $\log _{10}(\Lambda / \mathrm{GeV})$ & $A(\Lambda)$ & $B(\Lambda)$ \\
\hline 4 & 1.219 & 157 \\
5 & 1.533 & 186 \\
7 & 1.805 & 212 \\
9 & 1.958 & 230 \\
11 & 2.071 & 245 \\
13 & 2.155 & 258 \\
15 & 2.221 & 268 \\
19 & 2.278 & 277 \\
\hline
\end{tabular}

Table: Coefficients $A(\Lambda)$ and $B(\Lambda)$ of Eq. (28). 
The dependence on $\alpha_{S}\left(M_{Z}\right)$ is illustrated in Fig. 8, where we have taken [27]

$$
\alpha_{S}\left(M_{Z}\right)=0.124 \pm 0.006
$$

fixed $\Lambda$ to its maximum physically interesting value of $10^{19} \mathrm{GeV}$, and represented the lower bound on $M_{H}$ for the central value of $\alpha_{S}$ in (29) (diagonal thick solid line) and the two extreme values (diagonal thick dashed lines). A fit to these lines, accurate to $1 \mathrm{GeV}$ for $M_{H}>60 \mathrm{GeV}$, as those in (28), is given by,

$$
M_{H} / \mathrm{GeV}=\left[2.278-4.654\left(\alpha_{S}-0.124\right)\right]\left(M_{t} / \mathrm{GeV}\right)-277 .
$$

We have also shown in the plot the bounds corresponding to the requirement of absolute stability [8] (diagonal thin lines) and, for the sake of comparison, the absolute upper bound in the MSSM [15] corresponding to the same values of $\alpha_{S}$ and to $\Lambda_{\mathrm{SUSY}}=$ $1 \mathrm{TeV}$.

\section{Conclusions}

We have obtained absolute lower bounds on the SM Higgs mass $M_{H}$ as a function of the top-quark mass $M_{t}$, and the scale $\Lambda$ beyond which the SM is no longer valid, from the requirement of no decay by thermal fluctuations from the metastable minimum at the origin to the true (deep) minimum at large values of the field. The bounds from the similar requirement of no tunnelling by quantum fluctuations from the electroweak minimum at zero-temperature are always weaker than the former ones. For completeness we present them in Fig. 9, which should be compared with the solid lines in Fig. 7.

Now we will comment on the accuracy of our results. There are two types of uncertainties: theoretical and experimental. We find that the former are negligible as compared to the latter. The theoretical uncertainties, leaving apart the very precise treatment of the numerical analysis, include the definition of the critical probability, and the possible gauge dependence of the result. As for the former, we have seen in Fig. 5 that the result is completely insensitive to the precise definition of the critical probability. Any value of $\mathcal{O}(1)$ would give the same result \. A related uncertainty comes from the pre-factor $\omega$ in the probability rate (16). We expect this uncertainty to be comparable in size to that associated with the definition of the critical probability, and thus negligible when translated into an error in the determination of the Higgs mass. As for the gauge dependence, we expect it to affect our results very little. In fact, as can be seen from Figs. 1 and 4, the total effective potential at finite temperature is totally dominated by the thermal correction, for $\phi<\phi_{B}(0)$. The thermal correction (10), coming from gauge bosons and the top-quark, as well as the Debye masses (14), are gauge-independent [19], while all the gauge dependence is encoded in the one-loop contribution from the Higgs and Goldstone bosons to (10), which we have neglected,

\footnotetext{
${ }^{7}$ In fact the uncertainty in the determination of the Higgs mass from the effective potential at zero temperature, even if bounded by $\lesssim 1 \mathrm{GeV}$ in our treatment [8], is much greater than the uncertainty from the definition of the critical probability.
} 
since it is numerically irrelevant 8 . Finally we should mention that the very definition of the cutoff, or new physics scale, $\Lambda$ has, itself, a fudge factor which increases when the value of $\Lambda$ decreases. This is associated with the existence of threshold effects at the scale $\Lambda$, necessary to match the SM below $\Lambda$ with the new physics beyond $\Lambda$. In practice this effect should affect negligibly our results for high values of the scale $\Lambda$.

The experimental uncertainties come from the uncertainty in the determination of $\alpha_{S}\left(M_{Z}\right)$, which we will take as in (29), and the uncertainty in the (future) measurement of $M_{t}$. Normalizing the latter as $M_{t}=176 \pm 13 \mathrm{GeV}$ [9], we can write the uncertainty in the presented lower bound for $\Lambda=10^{19} \mathrm{GeV}$, as

$$
\Delta M_{H}=4.91\left(\frac{\Delta \alpha_{S}}{0.006}\right)\left(\frac{M_{t}}{176}\right)+29.6\left(\frac{\Delta M_{t}}{13}\right)
$$

where all masses are expressed in $\mathrm{GeV}$. Let us notice that the uncertainty in (31]) gets reduced for $\Lambda<10^{19} \mathrm{GeV}$. In particular for $\Lambda=10^{4} \mathrm{GeV}$ the factor 4.91 in (31) becomes $\sim 1$ and the factor 29.6 becomes $\sim 17$. A quick glance at (31) shows that only a very precise determination of $\alpha_{S}$ and $M_{t}$ can help in reducing the uncertainty on the bound of $M_{H}$.

On the other hand, one can easily extract information on the scale of new physics $\Lambda$ from a possible measurement of the Higgs mass at LEP-200 and an experimental lower bound on $M_{t}$. In fact, from Eq. (30) and the shape of bounds in Fig. 6 as functions of $\Lambda$ we can deduce that the measurement of $M_{H}$ would translate into an upper bound on the scale of new physics provided that $M_{t}$ satisfies 9

$$
M_{t}>\frac{M_{H}}{2.25}+123
$$

where all masses are in $\mathrm{GeV}$. That is, from (32) and the present bound on $M_{H}$, we obtain that $M_{t}>152 \mathrm{GeV}$ is necessary for a future Higgs-mass measurement to imply an upper bound on the scale of new physics. Non-detection of the Higgs at LEP-200, i.e. $M_{H}>90 \mathrm{GeV}$, would imply similarly $M_{t}>163 \mathrm{GeV}$ as a necessary condition to obtain an upper bound on the scale of new physics from a future Higgs-mass measurement. On the other hand, assuming experimental values for the Higgs and top-quark masses, $M_{t}^{\exp } \pm \Delta M_{t}^{\exp }$ and $M_{H}^{\exp } \pm \Delta M_{H}^{\text {exp }}$, the condition for an upper bound $\Lambda_{\text {max }}$ on new physics is provided by Eq. (32), with

$$
M_{t}=M_{t}^{\exp }-\Delta M_{t}^{\exp }
$$

and

$$
M_{H}=M_{H}^{\exp }+\Delta M_{H}^{\exp }
$$

and the precise value of $\Lambda_{\max }$ is given by the intersection of (33) and (34) in Fig. 6 .

Finally, notice that the Higgs mass measurement might serve (depending on the top mass) to disentangle between the SM, with a cutoff at $\Lambda=10^{19} \mathrm{GeV}$, and the MSSM

\footnotetext{
${ }^{8}$ Neglecting the scalar sector in radiative corrections is a normal procedure for analysing the electroweak phase transition [28]. In our case this approximation is especially justified since $\lambda \ll 1$ in the region near the stable (unphysical) minimum.

${ }^{9}$ Notice that we have used in (32) the $1 \sigma$ bound on $\alpha_{S}\left(M_{Z}\right)$ in (29), i.e. $\alpha_{S}<0.130$.
} 
with $\Lambda_{\text {SUSY }} \gg M_{Z}$ (in which case the couplings of the lightest Higgs are indistinguishable 29] from the couplings of the SM Higgs). In Fig. 8 we have plotted the upper bound on the light-Higgs mass in the MSSM for $\Lambda_{\text {SUSY }} \lesssim 1 \mathrm{TeV}$. We can see that only if $M_{t} \gtrsim 180 \mathrm{GeV}$ there is a mass gap between the MSSM and the SM, and measurement of $M_{H}$ will always exclude at least one of these models. However, for $M_{t} \lesssim 180 \mathrm{GeV}$ there is a large overlapping region where both models would be indistinguishable.

\section{Acknowledgements}

We thank W. Buchmüller and M. Shaposhnikov for useful comments and discussions, and J. Ignatius and J.M. Moreno for their help in handling critical bubbles. 


\section{References}

[1] M. Lindner, Z. Phys. C31 (1986) 295

[2] M. Sher, Phys. Rep. 179 (1989) 273

[3] M. Lindner, M. Sher and H.W. Zaglauer, Phys. Lett. B228 (1989) 139

[4] M. Sher, Phys. Lett. B317 (1993) 159; Addendum: Phys. Lett. B331 (1994) 448

[5] C. Ford, D.R.T. Jones, P.W. Stephenson and M.B. Einhorn, Nucl. Phys. B395 (1993) 17

[6] G. Altarelli and I. Isidori, Phys. Lett. B337 (1994) 141

[7] M. Quirós, invited talk presented at the Workshop on Physics from the Planck Scale to the Electroweak Scale, University of Warsaw, Poland, 21-24 September 1994; preprint CERN-TH.7507/94 hep-ph/9411403

[8] J.A. Casas, J.R. Espinosa and M. Quirós, Phys. Lett. B342 (1995) 171

[9] F. Abe et al., CDF Collaboration, Phys. Rev. D50 (1994) 2966; Phys. Rev. Lett. 73 (1994) 225; preprint FERMILAB-PUB-95/022-E (2 March 1995)

[10] S. Abachi et al., D0 Collaboration, Phys. Rev. Lett. 72 (1994) 2138; preprint FERMILAB-PUB-354/E, Phys. Rev. Lett. 72 (1995) to appear; preprint FERMILAB-PUB-95/028-E (3 March 1995)

[11] Z. Kunszt and W.J. Stirling, Phys. Lett. B262 (1991) 54

[12] G. Anderson, Phys. Lett. B243 (1990) 265

[13] P. Arnold and S. Vokos, Phys. Rev. D44 (1991) 3620

[14] For a recent review, see, e.g.: M. Quirós, Helv. Phys. Acta 67 (1994) 451

[15] J.A. Casas, J.R. Espinosa, M. Quirós and A. Riotto, Nucl. Phys. B436 (1995) 3

[16] B. Kastening, Phys. Lett. B283 (1992) 287;

M. Bando, T. Kugo, N. Maekawa and H. Nakano, Phys. Lett. B301 (1993) 83;

Prog. Theor. Phys. 90 (1993) 405

[17] N. Gray, D.J. Broadhurst, W. Grafe and K. Schilcher, Z. Phys. C48 (1990) 673

[18] L. Dolan and R. Jackiw, Phys. Rev. D9 (1974) 3320;

S. Weinberg, Phys. Rev. D9 (1974) 3357

[19] J.I. Kapusta, Finite-temperature field theory (Cambridge University Press, 1989)

[20] D.J. Gross, R.D. Pisarski and L.G. Yaffe, Rev. Mod. Phys. 53 (1981) 43 
[21] M.E. Carrington, Phys. Rev. D45 (1992) 2933;

M.E. Shaposhnikov, Phys. Lett. B277 (1992) 324 and (E) Phys. Lett. B282 (1992) 483 ;

M. Dine, R.G. Leigh, P. Huet, A. Linde and D. Linde, Phys. Lett. B283 (1992) 319 and Phys. Rev. D46 (1992) 550;

J.R. Espinosa and M. Quirós, Phys. Lett. B305 (1993) 98;

J.R. Espinosa, M. Quirós and F. Zwirner, Phys. Lett. B314 (1993) 206;

C.G. Boyd, D.E. Brahm and S.D. Hsu, Phys. Rev. D48 (1993) 4963;

P. Arnold and O. Espinosa, Phys. Rev. D47 (1993) 3546;

W. Buchmüller, T. Helbig and D. Walliser, Nucl. Phys. B407 (1993) 387

[22] J.S. Langer, Ann. Phys. (NY) 41 (1967) 108 and 54 (1969) 258

[23] A.D. Linde, Phys. Lett. B70 (1977) 306; Phys. Lett. B100 (1981) 37; Nucl. Phys. B216 (1983) 421

[24] J. Kripfganz, A. Laser and M.G. Schmidt, Nucl. Phys. B433 (1995) 467;

J. Baacke, preprint DO-TH-95/04 hep-ph/9503350

[25] A.H. Guth and E. Weinberg, Phys. Rev. D23 (1981) 876

[26] P. Janot, talk given at the First Plenary Meeting of the Physics at LEP II Workshop, CERN, 3 February 1995

[27] See, e.g.: P. Langacker, preprint UPR-0624T hep-ph/9408310

[28] G.W. Anderson and L.J. Hall, Phys. Rev. D45 (1992) 2685

[29] H. Haber, invited talk presented at the Workshop on Physics from the Planck Scale to the Electroweak Scale, University of Warsaw, Poland, 21-24 September, 1994; preprint SCIPP 94/39 (December 1994) 


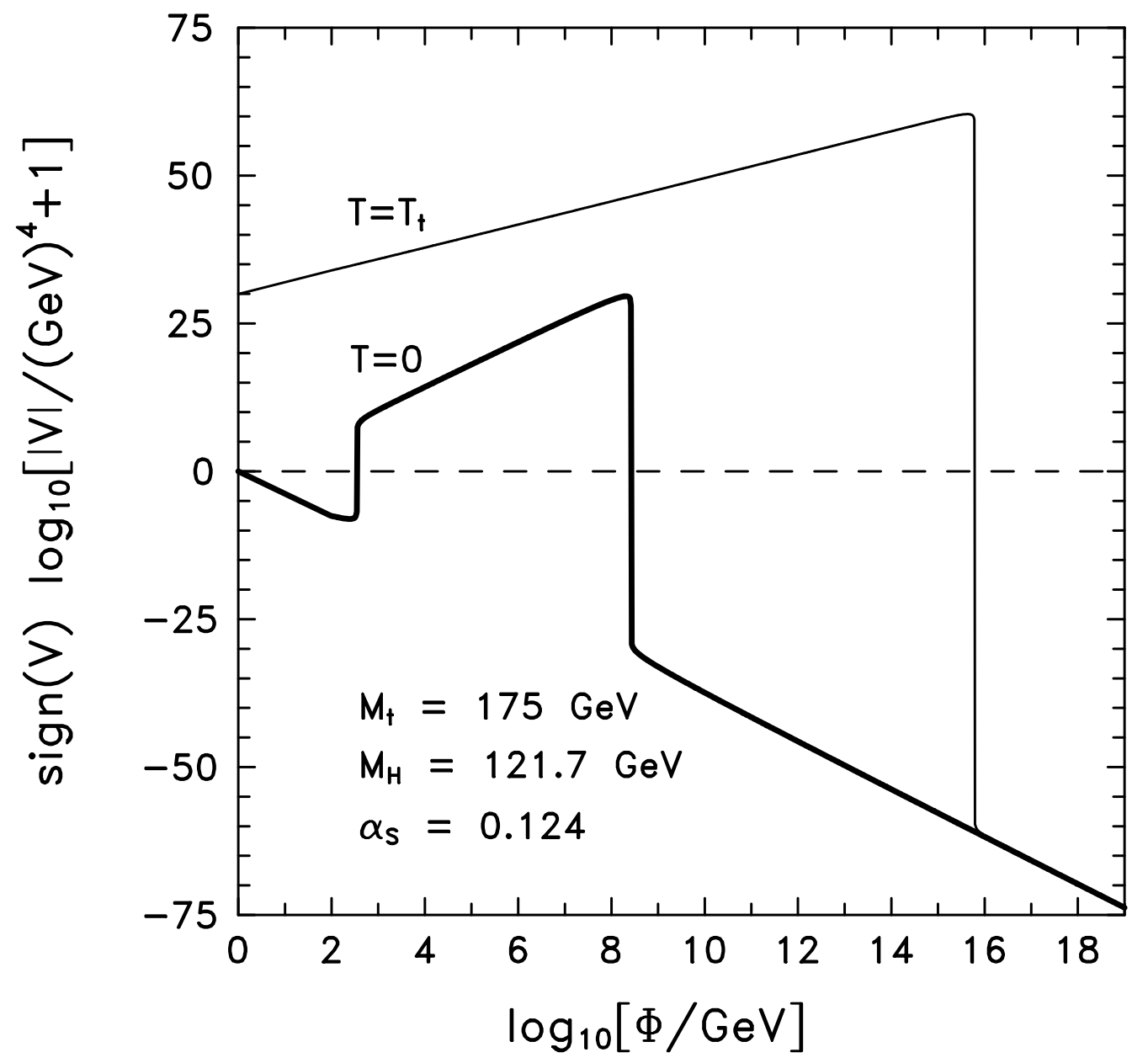

Figure 1: Plot of the effective potential for $M_{t}=175 \mathrm{GeV}, M_{H} \sim 122 \mathrm{GeV}$ at $T=0$ (thick solid line) and $T=T_{t}=2.5 \times 10^{15} \mathrm{GeV}$ (thin solid line). 


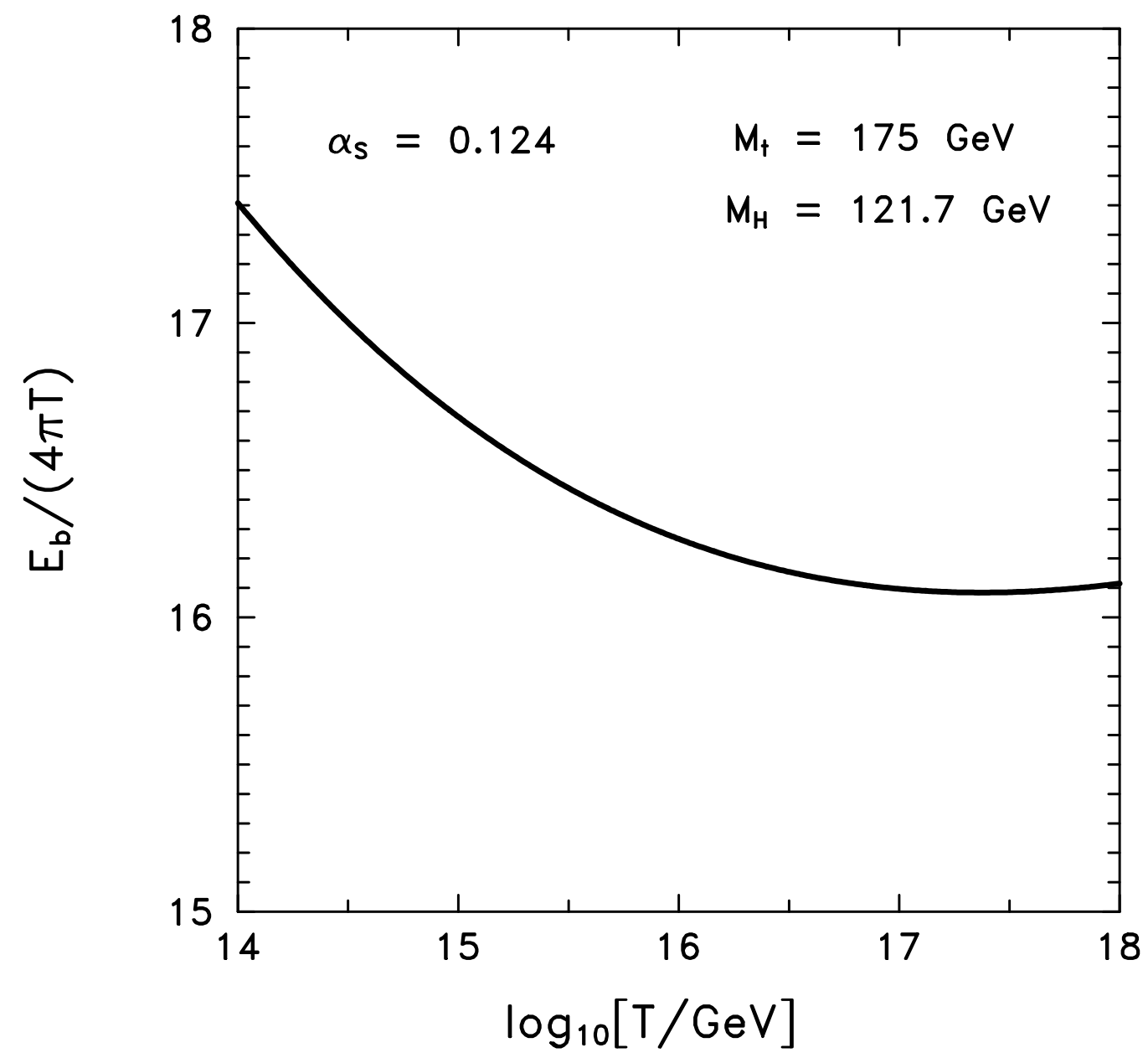

Figure 2: Plot of $E_{b}$, the energy of the critical bubble, as a function of the temperature for the same values of $M_{t}$ and $M_{H}$ as in Fig. 1. 


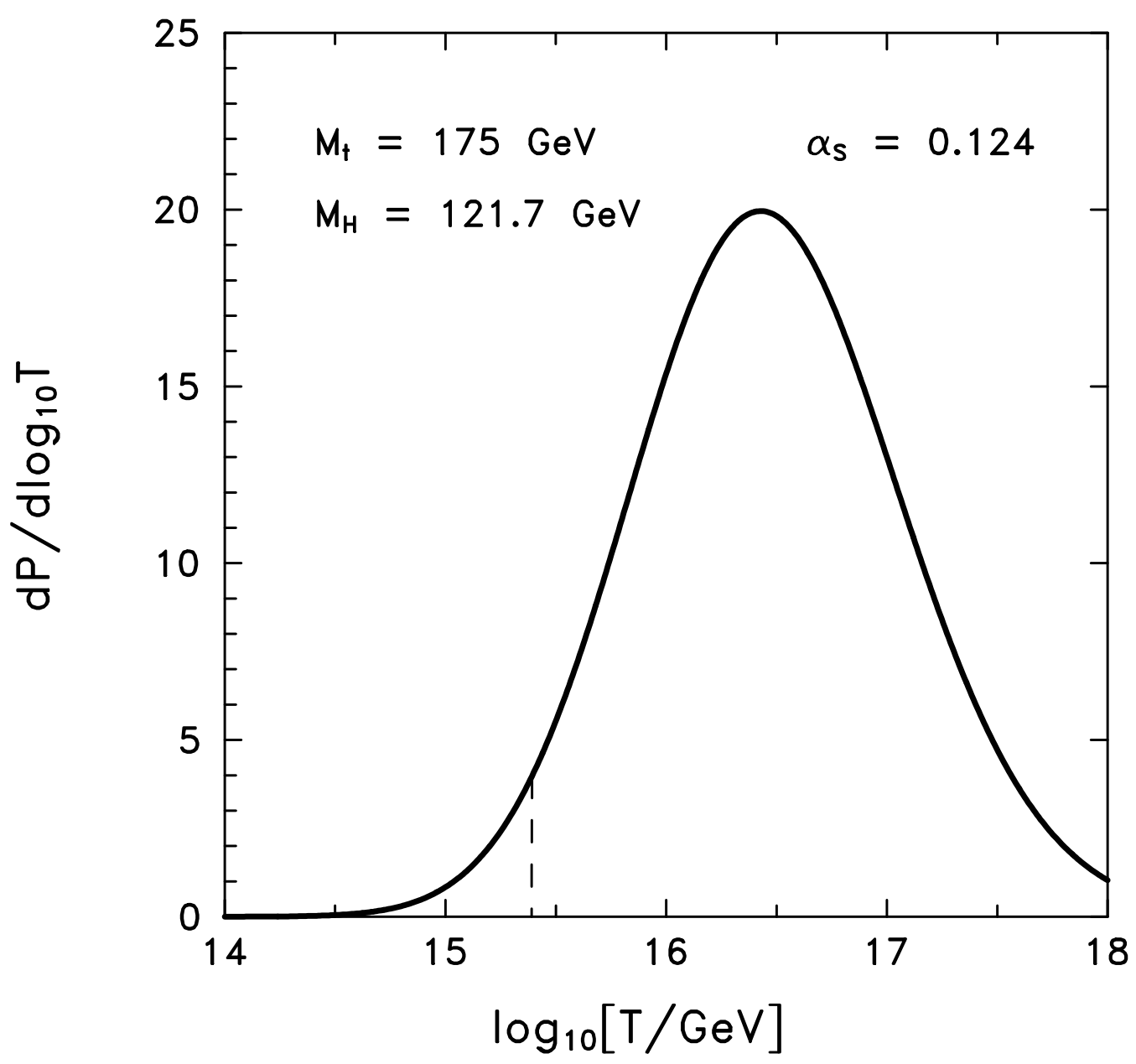

Figure 3: Plot of $d P / d \log _{10} T$ as a function of the temperature for the same values of $M_{t}$ and $M_{H}$ as in Fig. 1. The temperature $T_{t}=2.5 \times 10^{15} \mathrm{GeV}$ at which the integrated probability is equal to 1 is indicated with a dashed line. 


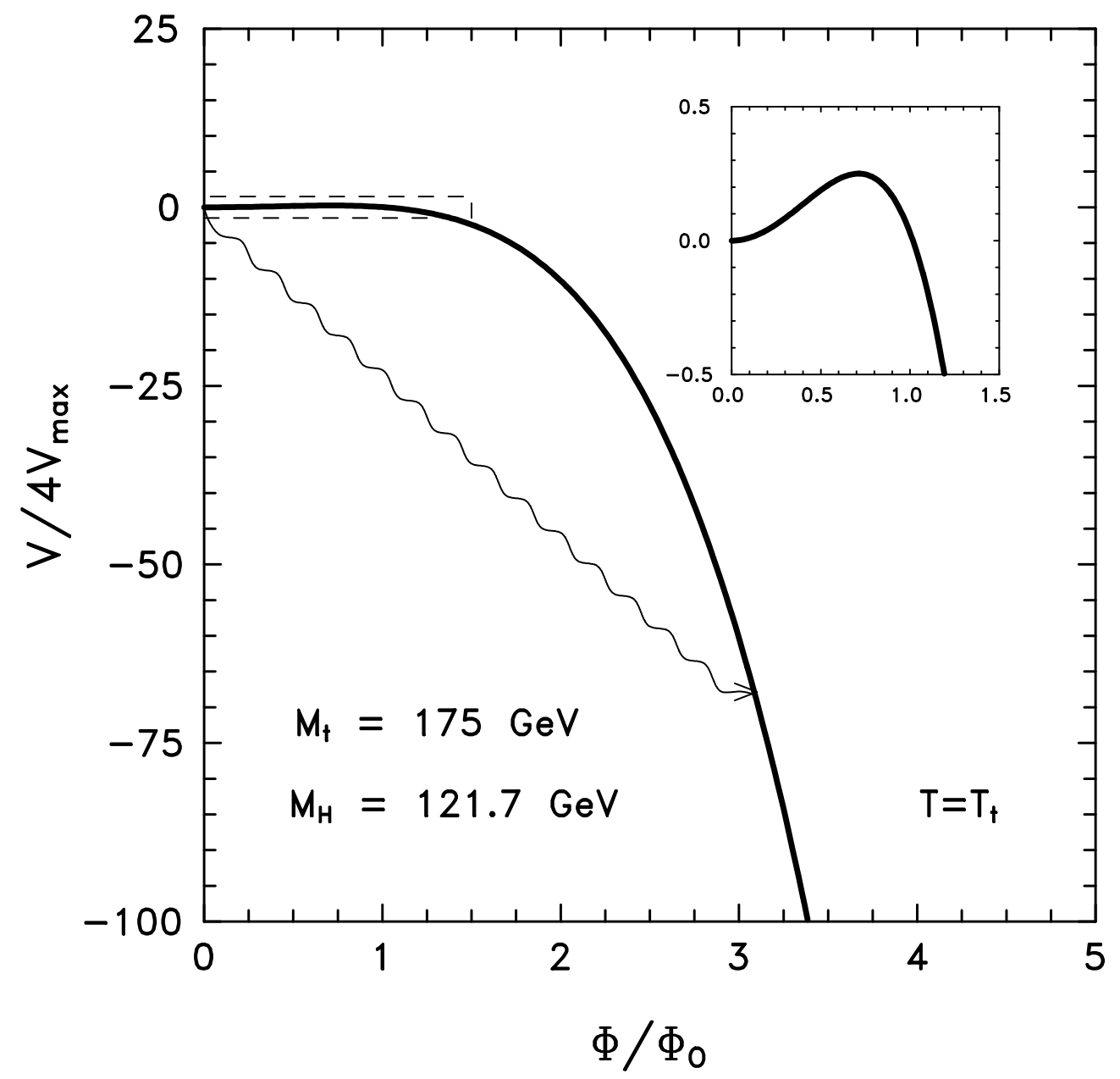

Figure 4: Plot of the effective potential at $T_{t}=2.5 \times 10^{15} \mathrm{GeV}$, for the same values of $M_{t}$ and $M_{H}$ as in Fig. 1, normalized with respect to its maximum value, as a function of $\phi$, arbitrarily normalized with $\phi_{0}=6.0 \times 10^{15} \mathrm{GeV}$. The arrow indicates the value of the bounce solution $\phi_{B}(0)$. 


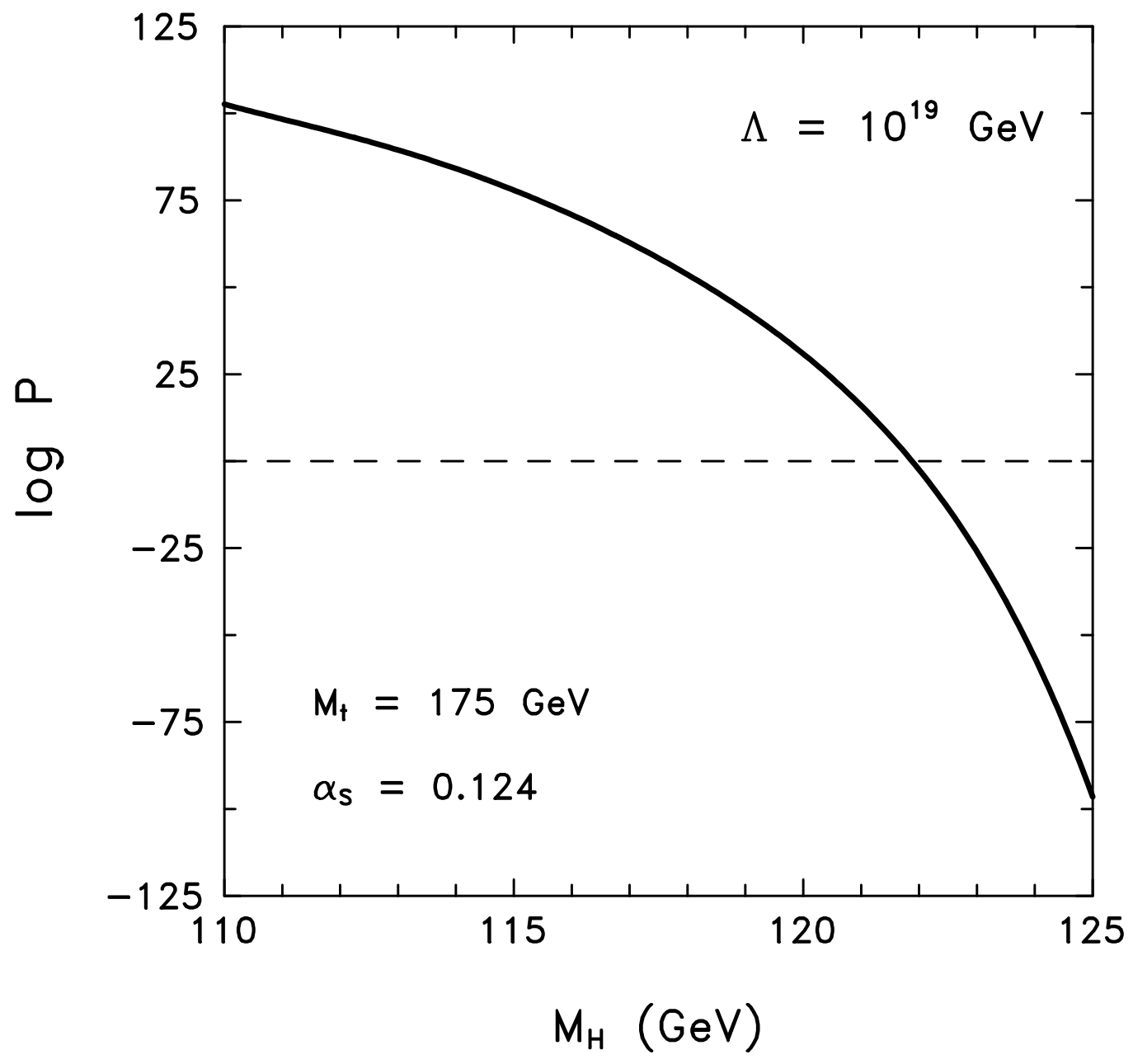

Figure 5: Plot of the logarithm of the total probability $(\log P)$ as a function of $M_{H}$ for $M_{t}=175 \mathrm{GeV}$. 


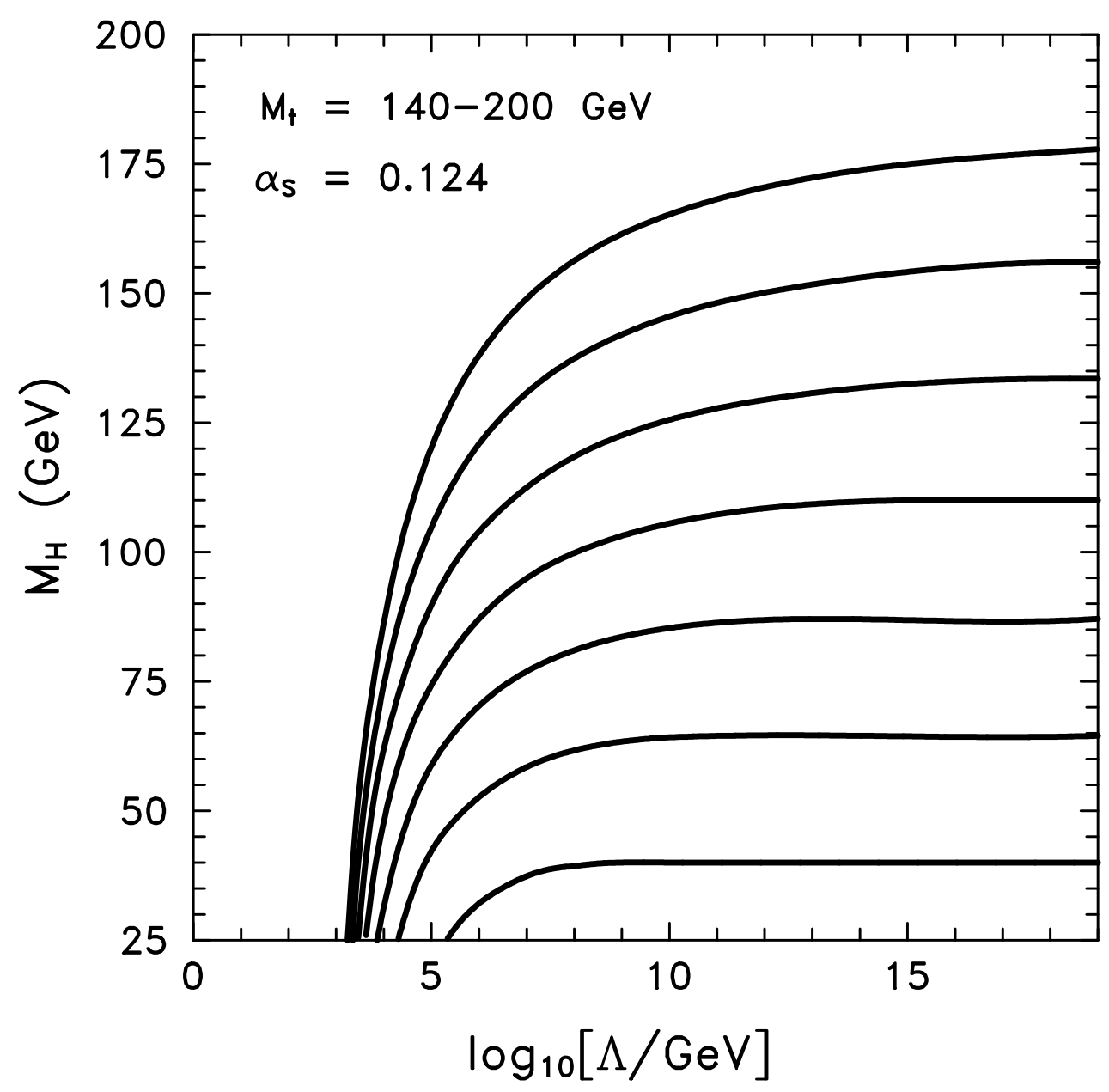

Figure 6: Lower bounds on $M_{H}$ as a function of the SM cutoff $\log _{10}[\Lambda / \mathrm{GeV}]$ for $\alpha_{S}\left(M_{Z}\right)=0.124$ and $M_{t}$ from $140 \mathrm{GeV}$ (lower curve) to $200 \mathrm{GeV}$ (upper curve), step $=10 \mathrm{GeV}$. 


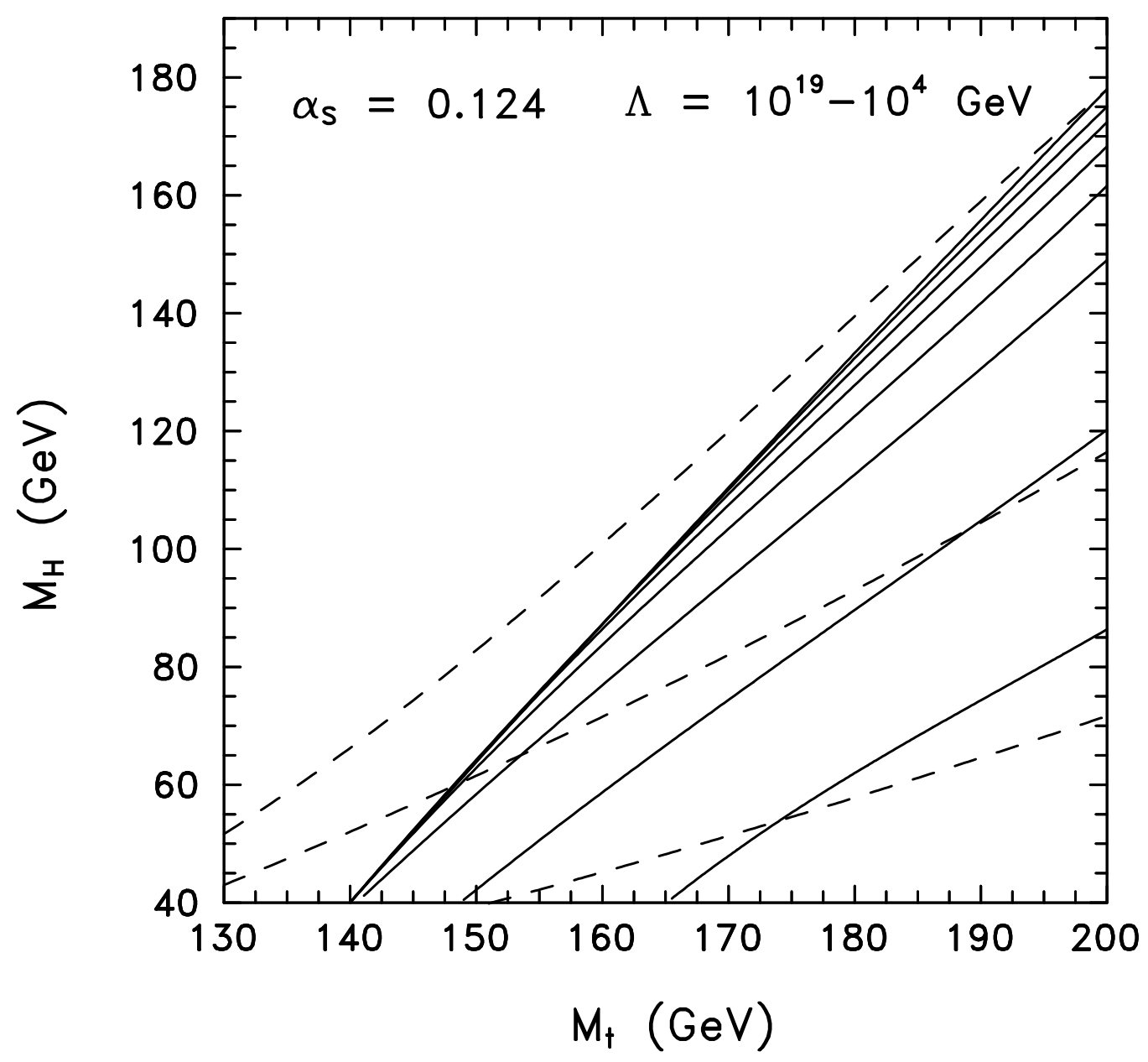

Figure 7: Lower bounds on $M_{H}$ as a function of $M_{t}$ for $\alpha_{S}\left(M_{Z}\right)=0.124$ and $\Lambda=$ $10^{4} \mathrm{GeV}$ (lower solid line), $10^{5} \mathrm{GeV}, 10^{7} \mathrm{GeV}, 10^{9} \mathrm{GeV}, 10^{11} \mathrm{GeV}, 10^{13} \mathrm{GeV}, 10^{15} \mathrm{GeV}$ and $10^{19} \mathrm{GeV}$ (upper solid line). The dashed lines are the absolute stability bounds for $\Lambda=10^{3} \mathrm{GeV}$ (lower dashed line), $10^{4} \mathrm{GeV}$ and $10^{19} \mathrm{GeV}$ (upper dashed line). 


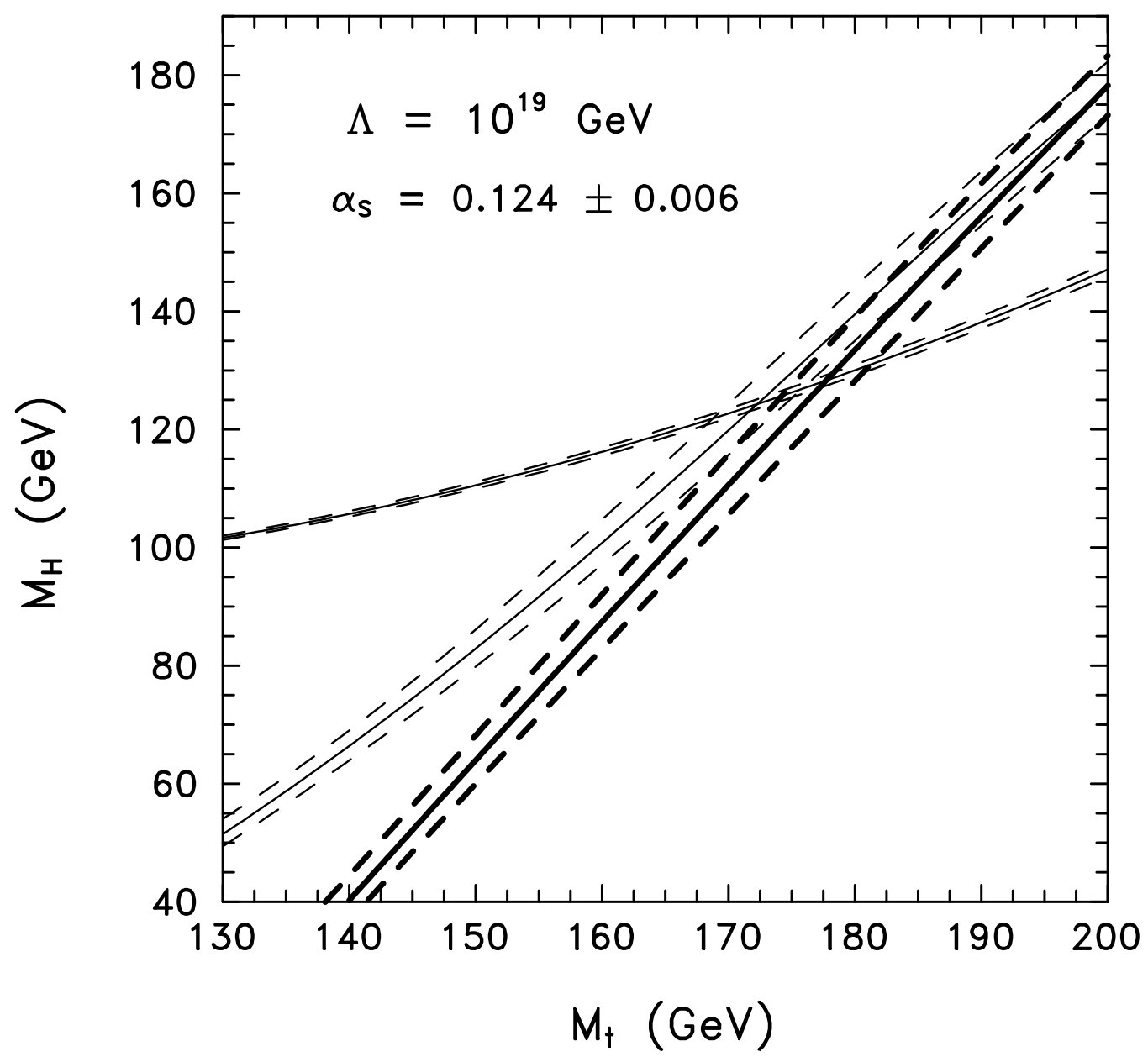

Figure 8: Diagonal lines: SM lower bound on $M_{H}$ (thick lines) as a function of $M_{t}$ for $\Lambda=10^{19} \mathrm{GeV}$ and $\alpha_{S}=0.124$ (solid), $\alpha_{S}=0.118$ (upper dashed), $\alpha_{S}=0.130$ (lower dashed). The corresponding bounds for the absolute stability requirement are the diagonal thin lines. Transverse (thin) lines: MSSM upper bounds on $M_{H}$ for $\Lambda_{\mathrm{SUSY}}=1 \mathrm{TeV}$ and $\alpha_{S}$ as in the diagonal lines. 


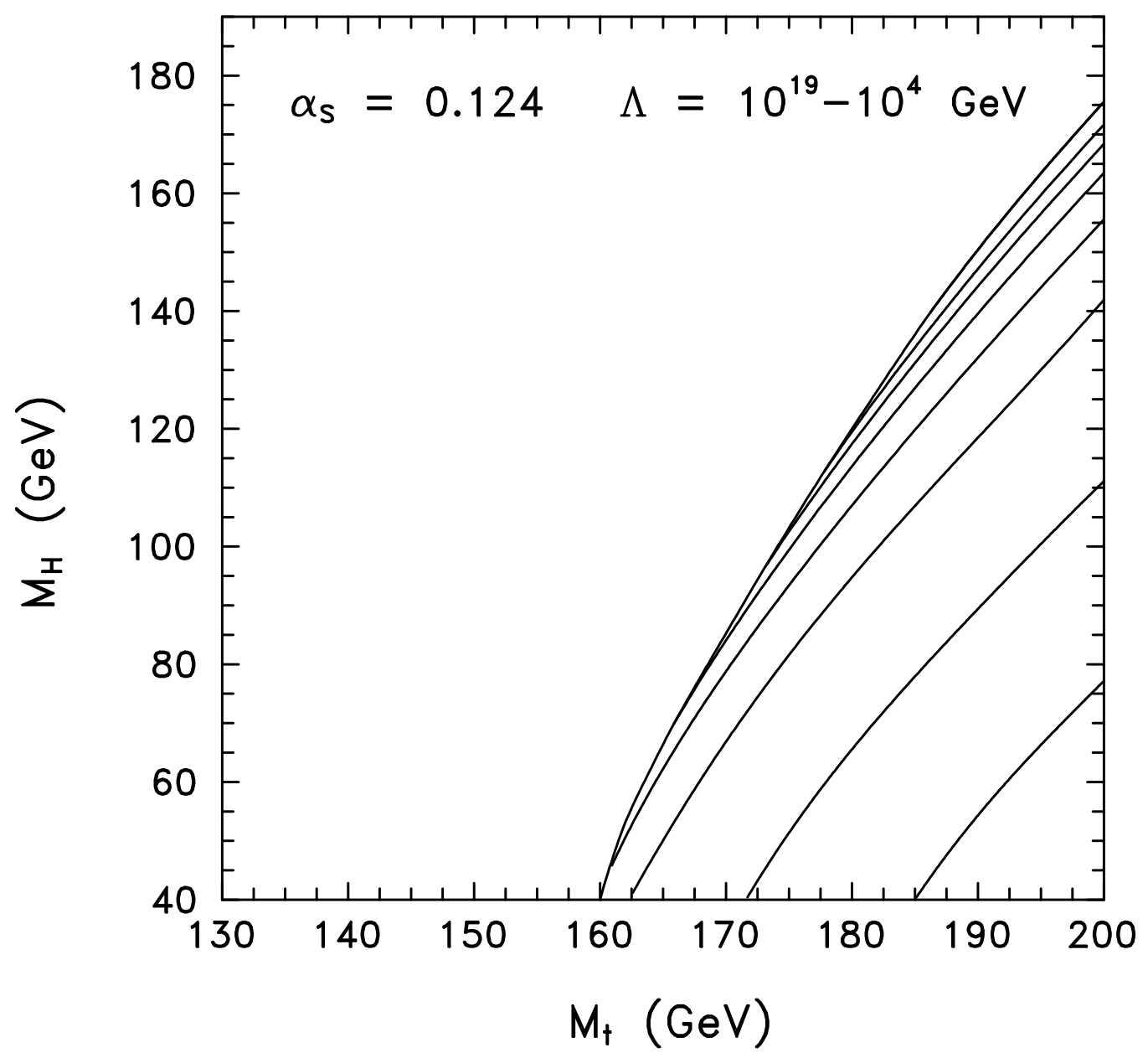

Figure 9: Lower bounds on $M_{H}$ as a function of $M_{t}$, for the values of $\alpha_{S}\left(M_{Z}\right)$ and $\Lambda$ shown as solid lines in Fig. 7, from the requirement of slow quantum tunnelling (compared with the present Universe expansion rate) from the electroweak minimum at $T=0$. 